\title{
Posterior parietal cortex activity predicts individual differences in visual short-term memory capacity
}

\author{
J. JAY TODD and RENÉ MAROIS \\ Vanderbilt University, Nashville, Tennessee
}

\begin{abstract}
Humans show a severe capacity limit in the number of objects they can store in visual short-term memory (VSTM). We recently demonstrated with functional magnetic resonance imaging that VSTM storage capacity estimated in averaged group data correlated strongly with posterior parietal/superior occipital cortex activity (Todd \& Marois, 2004). However, individuals varied widely in their VSTM capacity. Here, we examined the neural basis of these individual differences. A voxelwise, individualdifferences analysis revealed a significant correlation between posterior parietal cortex (PPC) activity and individuals' VSTM storage capacity. In addition, a region-of-interest analysis indicated that other brain regions, particularly visual occipital cortex, may contribute to individual differences in VSTM capacity. Thus, although not ruling out contributions from other brain regions, the individual-differences approach supports a key role for the PPC in VSTM by demonstrating that its activity level predicts individual differences in VSTM storage capacity.
\end{abstract}

Our ability to grab onto and hold in mind the information presented in a visual scene is extremely limited. For instance, subjects have great difficulty in detecting a gross change between two similar scenes if these scenes are separated in time or by another visual stimulus (Rensink, 2002; Rensink, O'Regan, \& Clark, 1997; Simons, 1996). Although the earliest stages of visual information processing are endowed with virtually unlimited processing capacities (Pashler, 1988; Phillips, 1974; Sperling, 1960), severe limits occur when the visual information is stored into visual working memory, which can at best accommodate four visual items (Cowan, 2001; Luck \& Vogel, 1997; Pashler, 1988; Vogel, Woodman, \& Luck, 2001). This bottleneck of information processing has significant real-life consequences, since it is considered to limit what information can be explicitly perceived and acted upon (Becker, Pashler, \& Anstis, 2000; Chun \& Potter, 1995; Jolicœur, Dell'Acqua, \& Crebolder, 2001; Rensink, 2002; Shapiro, Arnell, \& Raymond, 1997).

Due to the ubiquitous role of working memory in visual cognition, it is not too surprising that it has been associated with a large network of brain regions in both humans (J. D. Cohen et al., 1997; Courtney, Ungerleider, Keil, \& Haxby, 1997; Jonides et al., 1993; Linden et al., 2003; Munk et al., 2002; Pessoa, Gutierrez, Bandettini, \& Ungerleider, 2002; Sala, Rämä, \& Courtney, 2003) and nonhuman primates (Chafee \& Goldman-Rakic, 1998;

This work was supported by grants from the National Science Foundation and the National Institute of Mental Health to R.M. Correspondence should be addressed to R. Marois, Vanderbilt Vision Research Center, Department of Psychology, Vanderbilt University, 530 Wilson Hall, 111 21st Ave. S., Nashville, TN 37203 (e-mail: rene.marois@vanderbilt.edu).
Constantinidis \& Steinmetz, 1996; Funahashi, Bruce, \& Goldman-Rakic, 1989; Fuster, 1990; Goldman-Rakic, 1996; Miller \& Desimone, 1994; Quintana \& Fuster, 1999). Encompassing all the cortical lobes, this network can be loosely divided into regions that contribute primarily to Baddeley's (1986) central executive system and those regions involved in maintaining and storing the visual information that is manipulated by the executive system, the so-called visuospatial sketch pad (Baddeley \& Logie, 1999), or visual short-term memory (VSTM).

Executive processes, such as attentional selection, control, and manipulation of information, have been associated primarily with the frontal/prefrontal cortex (Bor, Duncan, Wiseman, \& Owen, 2003; Curtis \& D'Esposito, 2003; Sakai, Rowe, \& Passingham, 2002; Smith \& Jonides, 1999). By contrast, the simple storage of sensory information in VSTM or in auditory short-term memory is thought to involve more posterior regions (Paulesu, Frith, \& Frackowiak, 1993; Postle, Berger, \& D’Esposito, 1999; Rowe \& Passingham, 2001; Smith \& Jonides, 1998). In particular, substantial evidence indicates that the posterior parietal and inferior temporal regions are involved in the retention of visual information (Munk et al., 2002; Pessoa et al., 2002; Todd \& Marois, 2004). However, this anterior-posterior dissociation is likely not absolute, since the frontal/prefrontal cortex has also been involved in the simple maintenance of information (e.g., J. D. Cohen et al., 1997; Courtney, Petit, Maisog, Ungerleider, \& Haxby, 1998; Courtney et al., 1997; Curtis \& D’Esposito, 2003; Linden et al., 2003).

Thus, although there is considerable evidence that even the simple maintenance of information in VSTM recruits a vast network of brain regions (Linden et al., 
2003; Munk et al., 2002; Pessoa et al., 2002; Sala et al., 2003), the extent to which different nodes of this network process distinct components of VSTM is still not resolved (Pessoa et al., 2002). Furthermore, the neural basis of arguably the most distinctive characteristic of VSTM, its severely limited storage capacity, is poorly understood. Although a parametric load manipulation has been used in several studies to isolate brain regions associated with visual working memory (e.g., Braver et al., 1997; J. D. Cohen et al., 1997; Druzgal \& D’Esposito, 2003; Jha \& McCarthy, 2000; Leung, Gore, \& Goldman-Rakic, 2002; Linden et al., 2003), few have explored the neural substrates underlying the capacity constraints of working memory (e.g., Callicott et al., 1999; Duncan et al., 1999; Linden et al., 2003; Olesen, Westerberg, \& Klingberg, 2004; Vogel \& Machizawa, 2004).

In a recent study, we sought to identify the neural substrates associated with the capacity limit in storing a visual scene in VSTM (both object identities and locations; Todd \& Marois, 2004). In that study, even though subjects were shown from one to eight color objects (Figure 1), they were successful in storing only up to three or four of them in VSTM. More important, this VSTM storage capacity did not appear to be equally distributed across the neural network subserving visual working memory. Rather, activity in a particular brain region, the posterior parietal/superior occipital cortex (intraparietal sulcus and intraoccipital sulcus), was found to be tightly correlated with the limited number of objects stored in VSTM (Todd \& Marois, 2004). A much weaker VSTMcapacity-related signal was observed in the ventral occipital and frontal cortex. These results suggest that a key function of the posterior parietal cortex (PPC) in the neural network of visual working memory is the storage of visuospatial information.

The results of this study (Todd \& Marois, 2004) were based on a group average of VSTM load. That is, we sought to isolate brain regions that correlated with the average number of objects stored in VSTM at each load. However, an examination of individual storage capacity functions reveals a wide range of individual VSTM capacities (Figure 2). Using a formula modified from Pashler (1988) by Cowan (2001) to estimate VSTM capacity, the maximum number of objects maintained in VSTM was found to vary considerably between individuals, from 1.74 to 6.37 . This variation in capacity could arise from a wide spectrum of factors, ranging from differences in biology (e.g., genetics, age, and possibly gender), to task experience (e.g., Olesen et al., 2004; Raichle et al., 1994), and to differences in task strategies (Cornoldi \& Vecchi, 2003; Rypma, Berger, \& D’Esposito, 2002). But irrespective of what may be the underlying cause(s) for these individual differences in VSTM capacity, of most significance here is that a group average analysis does not capture the neural basis of individual differences, since it treats intersubject variability as error. If the activity of

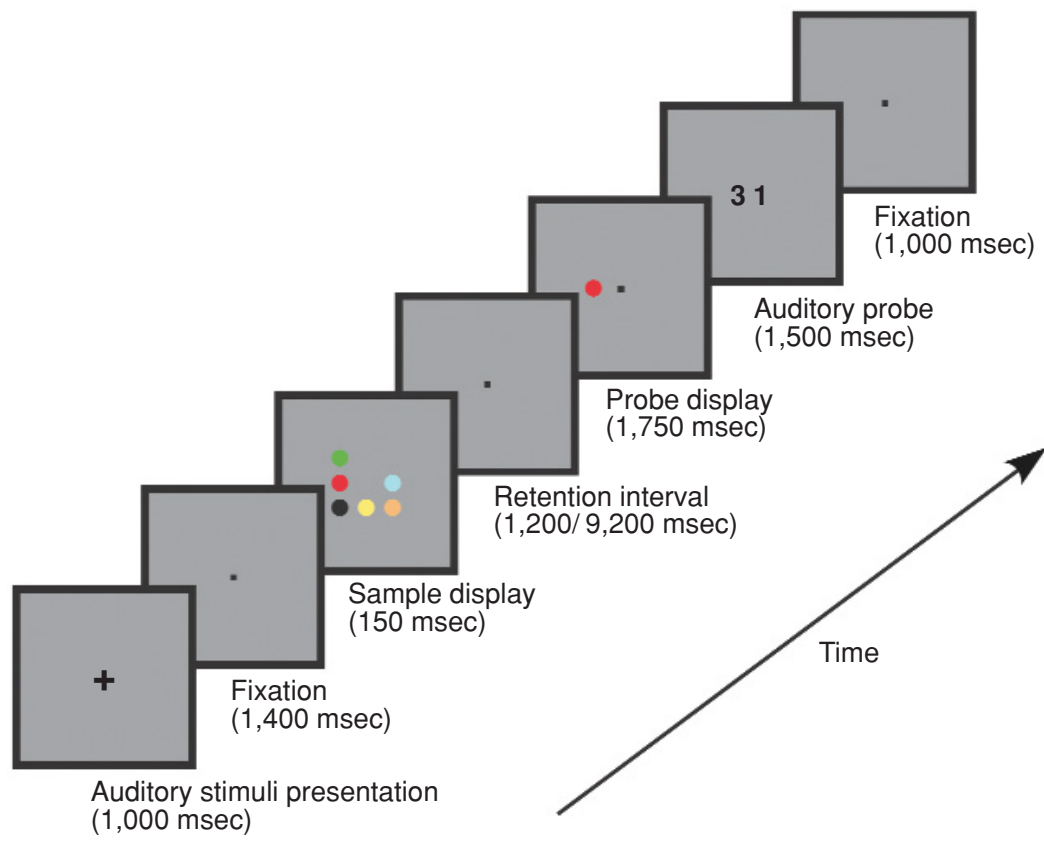

Figure 1. Trial design. Each trial began with the presentation of two digits, which were rehearsed subvocally throughout the trial. Next, a varying memory load of colored disks (one to eight) was presented. Following a brief retention interval $(1,200 \mathrm{msec}$ in Experiment 1, 9,200 msec in Experiment 2), the subjects determined whether a probe disk matched in color the disk presented at the same position in the sample display. Finally, a judgment was made regarding the two rehearsed digits. 


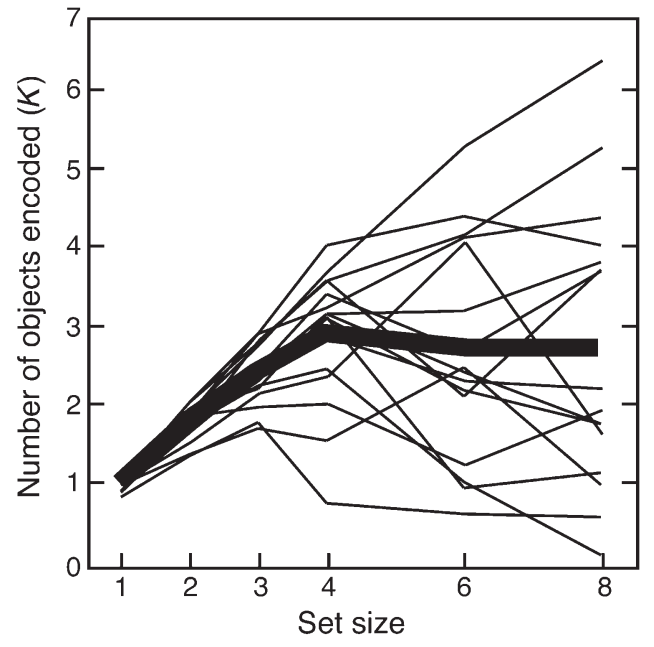

Figure 2. Individual visual short-term memory $K$ functions. The $K$ function for each individual (thin lines) is plotted by set size. The group mean $K$ function $(n=16)$ is represented by the thick line.

a brain region isolated with a group average analysis does not correlate with individual differences in performance, this would suggest that this brain region may contribute only a generic component to the cognitive process under investigation and that other brain regions account for the diversity of performance across subjects. On the other hand, a stronger case can be made about a brain region being involved in a specific cognitive process if that brain region not only is activated in a significant proportion of subjects, but also can account for the differences in performance between subjects. For example, in addition to demonstrating a role for the dorsal prefrontal cortex (DPFC) in the retrieval of information from auditory-verbal working memory (AVWM) in a group average analysis, Rypma and D'Esposito (1999) showed that individual differences in speed of information retrieval from AVWM was correlated with the level of DPFC activity. Thus, using both group and individualdifferences approaches, one can isolate a brain region that is activated across individuals in a task and, subsequently, identify its contribution to individual differences in performance.

The goal of the present study was to determine how an individual-differences approach can inform us about the neural basis of VSTM storage capacity. In particular, would an individual-differences approach provide converging evidence with the group average analysis for the importance of the PPC in VSTM storage capacity? Or would such individual-differences analysis instead point to other brain regions to account for intersubject variability? There is electrophysiological evidence in favor of the former possibility: Vogel and Machizawa (2004) showed that event-related potentials (ERPs) over posterior parietal and lateral occipital sites can predict individual subjects' VSTM capacity. Importantly, the source of this ERP signal is largely compatible with the poste- rior parietal/superior occipital locus of VSTM storage capacity isolated in the group average fMRI study (Todd $\&$ Marois, 2004).

To determine which neural substrates might capture individual differences in VSTM capacity, we adopted the logic of Vogel and Machizawa (2004). That is, if a brain region plays an important role in determining the amount of information held in VSTM, it is reasonable to expect a strong correlation between individual VSTM capacity estimates and the respective magnitude of activation in that brain region. In other words, individuals with large VSTM capacities would be expected to activate this brain region more than would individuals with smaller capacities. Thus, in the present study, we correlated each subject's brain activity at the set size at which they reached VSTM capacity with the number of objects they stored in VSTM at that same set size. We used this methodological approach in a voxelwise analysis in order to isolate any brain regions whose activity correlated with individual differences in VSTM capacity. We also applied this technique to a more sensitive region-of-interest (ROI) analysis in order to determine whether regions previously implicated in VSTM storage capacity on the basis of a group average analysis (Todd \& Marois, 2004) track individual differences in VSTM capacity.

\section{METHOD}

In the present study, the same data sets as those used in two extant functional magnetic resonance imaging (fMRI) experiments of VSTM load (Experiment 1, Todd \& Marois, 2004; Experiment 2, Todd, Fougnie, \& Marois, in press) were reanalyzed using an individualdifferences approach.

\section{Subjects}

The subjects were paid volunteers from the Vanderbilt community. Seventeen young adults ( 9 females; all right-handed) participated in Experiment 1, and 14 (8 females; 12 right-handed) volunteered for Experiment 2. All had normal or corrected-to-normal visual acuity and normal hearing and color vision. The experimental protocol was approved by and consent was received in accordance with the Institutional Review Board at the Vanderbilt University Medical Center.

\section{Task Design}

Experiment 1: Fast event-related fMRI study. A detailed description of the experimental method has been published elsewhere (Todd \& Marois, 2004). The subjects performed a parametric load manipulation of a delayed recognition task (Figure 1). A static display containing a varying number (one, two, three, four, six, or eight) of colored disks $\left(0.38^{\circ}\right.$ of visual angle), each of a different color (red, green, dark green, blue, light blue, black, white, brown, or yellow), was presented for $150 \mathrm{msec}$. The stimuli were presented at nine possible locations within an invisible $3 \times 3$ matrix $\left(1.38^{\circ}\right.$ square). After a $1,200-\mathrm{msec}$ retention period, a single colored disk was presented in a position previously occupied by a disk in the sample array. The subjects indicated by buttonpress whether the color of the probe disk was identical to the color of the sample disk at that position (right index finger, same; right middle finger, different). Half the trials were matched. To control for possible contamination of AVWM, the subjects performed a concurrent articulatory suppression task (Baddeley, 1986). At trial onset, two single-digit numbers were presented serially through headphones 
for $250 \mathrm{msec}$ each, followed by a 250 -msec blank interval and then by a 250 -msec auditory mask composed of superimposed forward and reversed versions of the individual sound files. Following the mask, there was a 1,400-msec period of fixation before the onset of the visual sample array. The subjects were instructed to rehearse the two digits throughout the trial. Following the visual probe response, the subjects indicated by buttonpress whether two visually presented digits were the same as those rehearsed throughout the trial.

Experiment 2: Slow event-related fMRI study. Fourteen young adults ( 8 females; 12 right-handed) volunteered for this experiment. The details of the experimental design have been published elsewhere (Todd et al., in press; Todd \& Marois, 2004). The experimental design was similar to that for the fast event-related fMRI experiment described above, with the following exceptions: The retention interval was extended from 1,200 to 9,200 msec (trial duration, $18 \mathrm{sec} ; 7$ trials/fMRI run), only two set sizes were employed in order to compensate for the lower number of trials acquired with a slow event-related paradigm, and there were no nonevent trials.

fMRI methods. fMRI methods have been described elsewhere (Todd \& Marois, 2004). Low- and three-dimensional (3-D) highresolution T1-weighted images were acquired using conventional techniques with a 3-T GE MRI scanner (GE Medical Systems, Milwaukee, WI). In each functional run, $220 \mathrm{~T} 2 *$-weighted echoplanar images were acquired in 19 axial slices $(7 \mathrm{~mm}$ thick, $3.75 \times 3.75 \mathrm{~mm}$ in-plane, 0 -mm skip, repetition time $(\mathrm{TR})=2,000 \mathrm{msec}$, echo time $=$ $25 \mathrm{msec}, \mathrm{FOV}=24 \mathrm{~cm}$, matrix $=64 \times 64$ ) covering the whole brain and prescribed parallel to the AC-PC line. Trial presentation was synchronized to TR onset by scanner trigger pulses. The stimuli were presented on an Apple G4 Macintosh using PsychToolBox for Matlab. The stimuli were back-projected onto a screen viewed by the supine subject in the MR scanner through a prism mirror. The fMRI methods for Experiment 2 were the same as those for Experiment 1.

\section{Data Analysis}

Behavioral analysis. The estimated number of objects stored in $\operatorname{VSTM}(K)$ was calculated using a formula developed by Pashler (1988) and modified by Cowan (2001). Here, $K=$ (hit rate + correct rejection rate -1$) N$, where $N$ is the number of objects presented in the sample array.

fMRI analysis. The fMRI data analysis was carried out with Brain Voyager 4.9.1 (Brain Innovation, Maastricht, The Netherlands). Preprocessing included intrasession image realignment, 3-D motion correction and correction for slice scan acquisition order using sinc interpolation, and linear trend removal.

Group statistical parametric map analysis. VSTM loadmodulated regions in Experiment 1 and for the right posterior middle frontal gyrus (see the Results section) were identified in statistical parametric maps (SPMs) of blood oxygen level dependent (BOLD) activation, using multiple regression analysis, with impulse regressors for each trial type (six set sizes and a no-event condition) convolved with a canonical hemodynamic response function (Boynton, Engel, Glover, \& Heeger, 1996). Regression coefficients were weighted for each set size by their respective group average $K$ value. The resulting maps from all the subjects were standardized into Talairach space (Talairach \& Tournoux, 1988) and were overlaid to create composite activation maps. The overall model fit was assessed with an $F$ statistic, using a random effects model, and voxels were considered significant if $p<.05$, corrected for multiple comparisons, using a cluster threshold of 6 voxels and spatial smoothing Gaussian kernel of $8.0 \mathrm{~mm}$ (FWHM; Ward, 2000). ROIs were isolated from the SPMs, and time courses for each memory load were extracted from these ROIs. Percentage of signal change of each time course was calculated using the nonevent time series as the baseline condition (Kourtzi \& Kanwisher, 2001).
Individual-differences analysis. Individual-differences analysis was carried out using both a voxel-based and an ROI-based approach. The voxelwise approach was used to identify regions that track individual differences in VSTM storage capacity. To isolate such regions, we first determined, for each subject, the maximum number of objects they could store $\left(K_{\max }\right)$ and the percentage of BOLD signal change (relative to no-event signal) obtained at the $K_{\max }$ set size. Since at set size 1 all the subjects had virtually the same $K$ value (mean $K=0.95$ ) but very different BOLD signal levels, we standardized brain activity across subjects by first subtracting the percentage of BOLD signal at set size 1 from the percentage of BOLD signal at set size $K_{\max }$. By using set size 1 activity as a baseline response for each individual, this transformation increased our sensitivity for detecting brain regions whose activity correlated with individual differences in VSTM capacity (Vogel \& Machizawa, 2004). For each individual, regressors were defined for set size $K_{\max }$ and set size 1 and were weighted by the individual's maximum $K$ value. We then probed for voxels whose activity covaried with the magnitude of the difference between set size $K_{\max }$ and set size 1 across individuals.

The ROI approach was used to determine whether brain regions previously implicated in VSTM storage capacity on the basis of a group average analysis (Todd \& Marois, 2004) contributed to individual differences in VSTM capacity. For each ROI, we correlated the individual differences in $K_{\max }$ with the individual differences in percentage of peak activation between set size $K_{\max }$ and set size 1 . Specifically, the volume corresponding to the peak of the hemodynamic response collapsed across all set sizes was first defined. Then, for each individual, the activation difference between set size $K_{\max }$ and set size 1 of the peak volume was computed, and these activation differences were subsequently correlated with each individual's $K_{\max }$. Significance for correlation analysis of the intraparietal sulcus/intraoccipital sulcus (IPS/IOS) ROI was set to $p<.05$, one-tail, on the basis of the a priori expectation of a positive linear relationship between VSTM capacity and brain activity. For consistency, the same statistical criteria were employed for all the ROIs. For Experiment 2, ROI analysis of individual differences in VSTM capacity ${ }^{1}$ was performed by first computing, for each individual, the difference in activation between set sizes 1 and 3 and regressing this activation difference with their corresponding $K$ value obtained at set size 3 across individuals. This analysis was performed separately for the encoding, maintenance, and retrieval phases of VSTM, using the peak volumes acquired $5.5-7.5,9.5-11.5$, and $15.5-17.5 \mathrm{sec}$ after stimulus presentation, respectively. Given the 9.2-sec-long retention interval, these peak time windows should provide largely uncontaminated estimates of activity for each of the three VSTM phases, particularly for the maintenance phase (Pessoa et al., 2002; Zarahn, Aguirre, \& D'Esposito, 1997).

Outliers were isolated and removed in ROIs using DFFITS $S_{i}$ with a cutoff threshold equal to \pm 1 (J. Cohen, P. Cohen, West, \& Aiken, 2003). At most, two outliers were removed from any given ROI.

\section{RESULTS}

\section{Voxel-Based Analyses}

The aim of the voxelwise analysis was to isolate brain regions whose activity correlated with individual differences in VSTM capacity. This SPM revealed a single region within the left IPS/IOS $[t(15)=4.42, p<.05$, corrected; Figure 3A]. The activated region largely overlapped the IPS/IOS region isolated in the group analysis (Todd \& Marois, 2004): group-based ROI Talairach coordinates, $x,-14$ to $-30 ; y,-81$ to $-58 ; z,+17$ to +49 ; individualbased ROI coordinates, $x,-17$ to $-29 ; y,-81$ to -61 ; $z,+21$ to +45 . The contralateral IPS/IOS was also acti- 
A
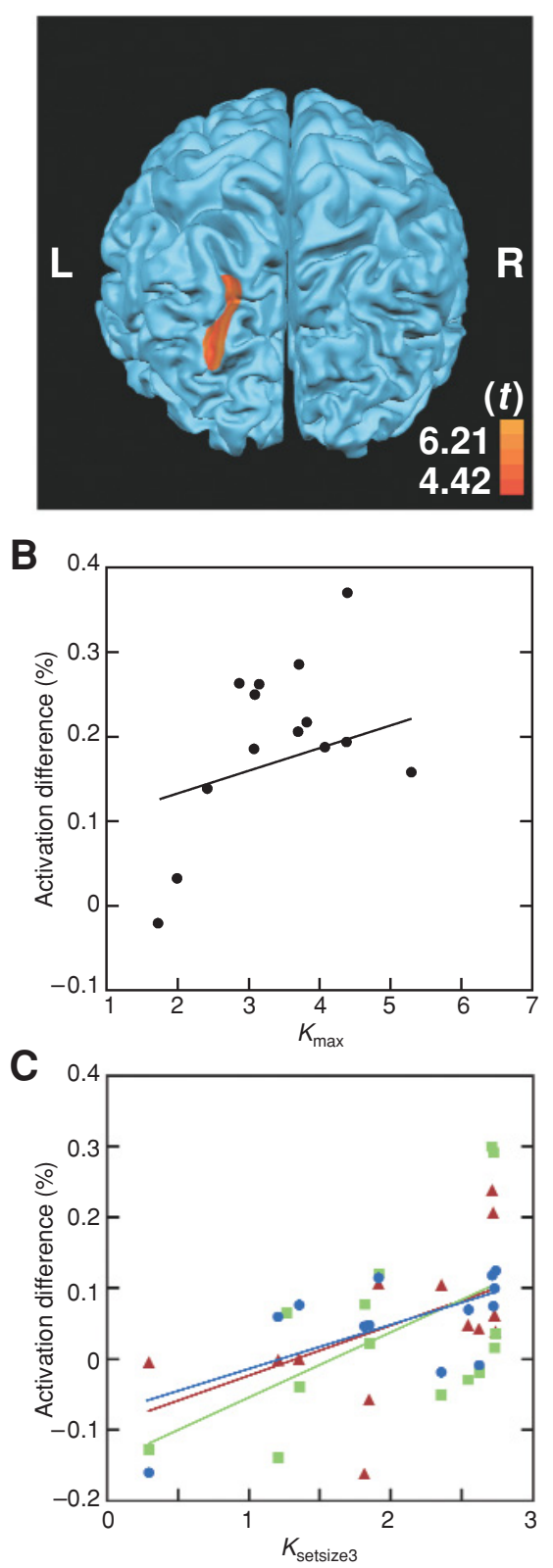

Figure 3. Intraparietal/intraoccipital regions of interest. (A) Statistical parametric map of individual differences in visual short-term memory (VSTM) storage capacity revealed activity along the left intraparietal sulcus/intraoccipital sulcus (IPS/IOS; $p<.05$, corrected). The peak voxel Talairach coordinates of this region are $x, y, z,-25,-77,+33$. The contralateral IPS/IOS is also activated when the threshold is reduced tenfold. (B) Correlation of individual $K_{\max }$ (VSTM storage capacity estimates) with the activation difference between set size $K_{\max }$ and set size 1 for Experiment 1. (C) Correlation of individual $K_{\text {setsize } 3}$ with the activation difference between set sizes 3 and 1 for the encoding (red), maintenance (green), and retrieval (blue) phases of VSTM in Experiment 2.

vated when the threshold was reduced tenfold. ROI analysis of this IPS/IOS region confirmed that individuals' $K_{\max }$ correlated with the activation difference between set size $K_{\max }$ and set size $1[r(12)=.56, p<.05$; Figure $3 \mathrm{~B}]$. This load-modulated response was not simply due to high- $K$ individuals demonstrating greater overall activation than low- $K$ individuals did (Vogel \& Machizawa, 2004), since each subject's BOLD signal was standardized at set size 1 . The response was also not due to scaling differences in activation across individuals, with subjects with greater memory capacity simply having greater overall activation range, since overall activation differences across set sizes did not correlate with individuals' $K_{\max }[r(14)=.36$, n.s.]. Importantly, the correlation between individuals' $K_{\max }$ and activity difference between set sizes $K_{\max }$ and 1 did not extend to supracapacity set sizes. That is, there was no correlation between individuals' $K_{\max }$ and the activation difference between set size 8 and $K_{\max }$ for the individuals whose $K_{\max }$ occurred at set sizes lower than $8[r(7)=-.19$, n.s.]. Analysis of the same ROI in Experiment 2 with extended retention interval revealed that the activity difference between set sizes 3 and 1 was correlated with individual VSTM capacities at set size 3 during maintenance $[r(12)=.54, p<$ $.05]$ and retrieval $[r(11)=.63, p<.05]$, and marginally so during encoding $[r(11)=.50, p=.08$; Figure $3 \mathrm{C}]$.

Taken together, these analyses indicate that a region of the IPS/IOS that largely overlaps with the PPC region isolated with a group-based approach to VSTM capacity (Todd \& Marois, 2004) tracks individual differences in VSTM capacity.

\section{ROI-Based Analyses}

In addition to the IPS/IOS, our group average study of VSTM load had revealed weak correlation between VSTM capacity and ventral occipital (VO) and anterior cingulate (AC) cortex activity (Todd \& Marois, 2004). Here, we examined the latter two ROIs to determine whether their activity reflects individual differences in VSTM capacity.

Ventral occipital ROI. Individual-differences analysis applied to Experiment 1, using the methods described for the IPS/IOS above, revealed that VO activity difference between set size $K_{\max }$ and set size 1 did not correlate with individuals' VSTM capacity $[r(14)=.32$, n.s.; Figure 4A $]$. However, individual-differences analysis for Experiment 2 indicated that $\mathrm{VO}$ activity difference between set sizes 3 and 1 correlated with individuals' VSTM capacity at set size 3 during maintenance $[r(12)=.63, p<$ .05 ; Figure $4 \mathrm{~B}]$, but not during encoding $[r(12)=-.02$, n.s.] or retrieval $[r(12)=.14$, n.s.]. Thus, a correlation between individuals' VSTM storage capacity and brain activity in VO is observed in the VSTM task with the long retention interval, but not with the short retention interval (Experiment 1).

Anterior cingulate ROI. In the AC, activation differences between set size $K_{\max }$ and set size 1 did not correlate significantly with individual capacity limit estimates $[r(13)=.35$, n.s.; Figure 5A]. Correspondingly, Experiment 2 revealed no significant correlation between AC activity and individual VSTM capacities during all three phases of VSTM [encoding, $r(11)=.17$, n.s.; maintenance, 

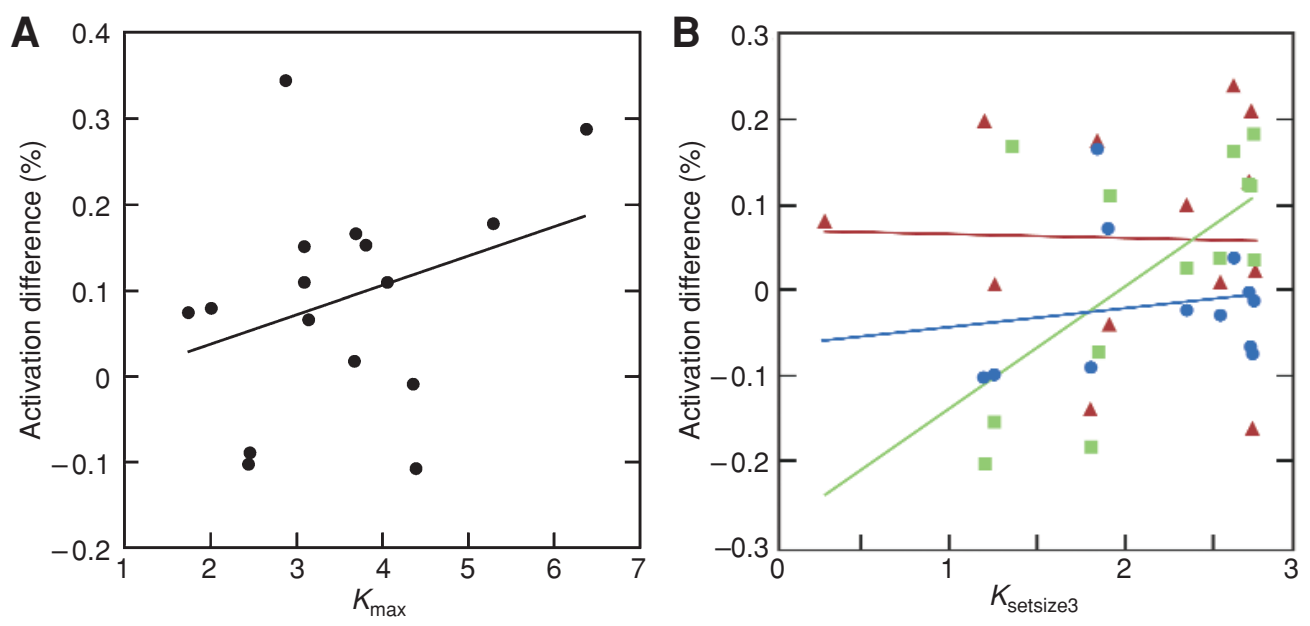

Figure 4. Bilateral ventral occipital region of interest. (A) Correlation of individual $K_{\max }$ with the activation difference between set size $K_{\max }$ and set size 1 in Experiment 1. (B) Correlation of individual $K_{\text {setsize3 }}$ with the activation difference between set sizes 3 and 1 for the encoding (red), maintenance (green), and retrieval (blue) phases of visual short-term memory in Experiment 2.

$r(12)=.42$, n.s.; retrieval, $r(12)=-.14$, n.s.; Figure 5B]. Thus, AC activity shows nonsignificant correlation trends with individual differences in VSTM capacity.

Right posterior middle frontal gyrus ROI. Our group average approach to VSTM capacity failed to reveal any regions of the frontal or prefrontal cortex that correlated with the number of objects stored in VSTM (Todd \& Marois, 2004). However, the role of these regions in visual working memory has been clearly established (Curtis \& D'Esposito, 2003; Druzgal \& D'Esposito, 2003; Leung et al., 2002; Linden et al., 2003; Postle, Stern, Rosen, \& Corkin, 2000; Sala et al., 2003). To determine whether an individual-differences analysis may better reveal a frontal/prefrontal contribution to VSTM storage capacity, we examined a region in the right posterior middle frontal gyrus (rpMFG; Figure 6A) that can be observed in the group average SPM of VSTM storage capacity (Todd \& Marois, 2004) when the statistical threshold $p$ was reduced 100 -fold prior to correction for multiple comparisons. A plot of the group average peak BOLD response at each VSTM set size (Figure 6B) revealed that the rpMFG response function was different from the group behavioral $K$ function (Figure 6B; Todd $\&$ Marois, 2004): Instead of leveling off around set size 3 or 4 , rpMFG response reached asymptote at set size 2 , a subcapacity VSTM set size. In addition, individualdifferences analysis revealed that the rpMFG activity difference between set size $K_{\max }$ and set size 1 did not cor-
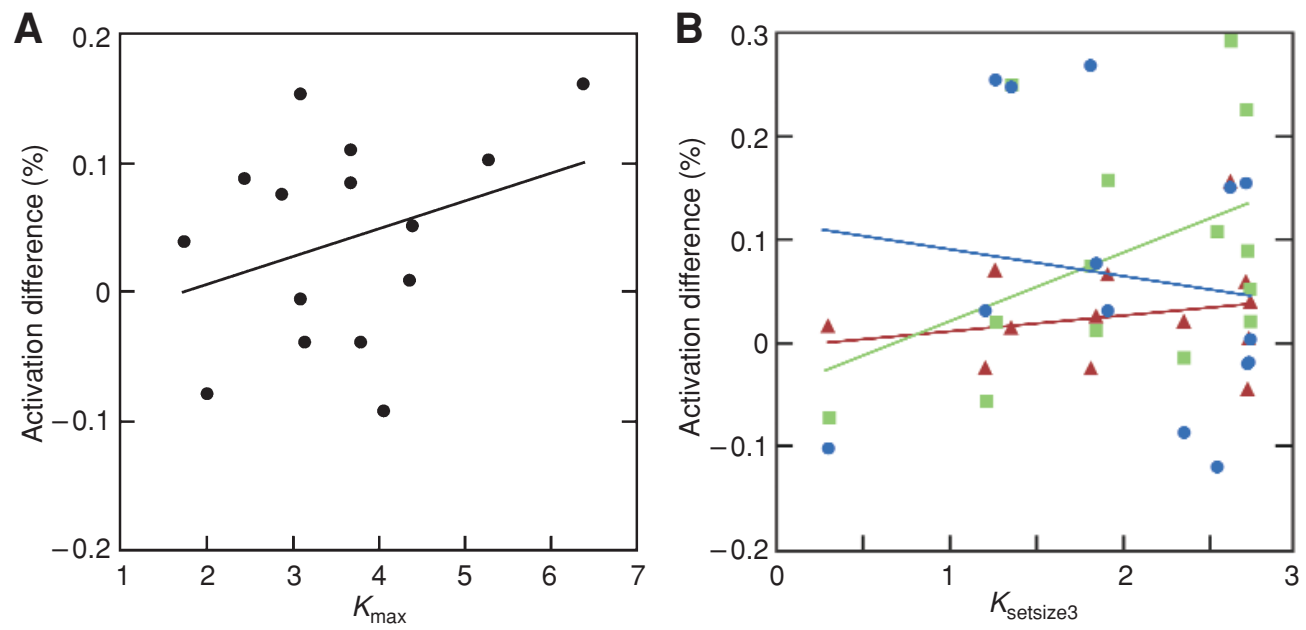

Figure 5. Anterior cingulate region of interest. (A) Correlation of individual $K_{\max }$ with the activation difference between set size $K_{\max }$ and set size 1 in Experiment 1. (B) Correlation of individual $K_{\text {setsizez }}$ with the activation difference between set sizes 3 and 1 for the encoding (red), maintenance (green), and retrieval (blue) phases of visual short-term memory in Experiment 2. 
A
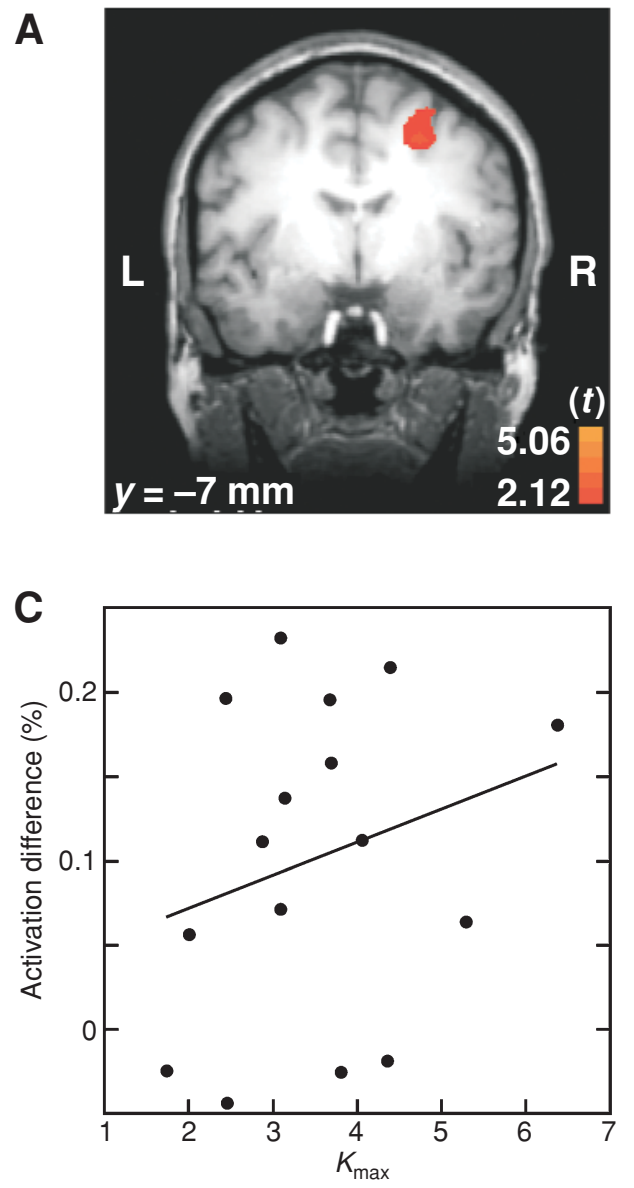

B
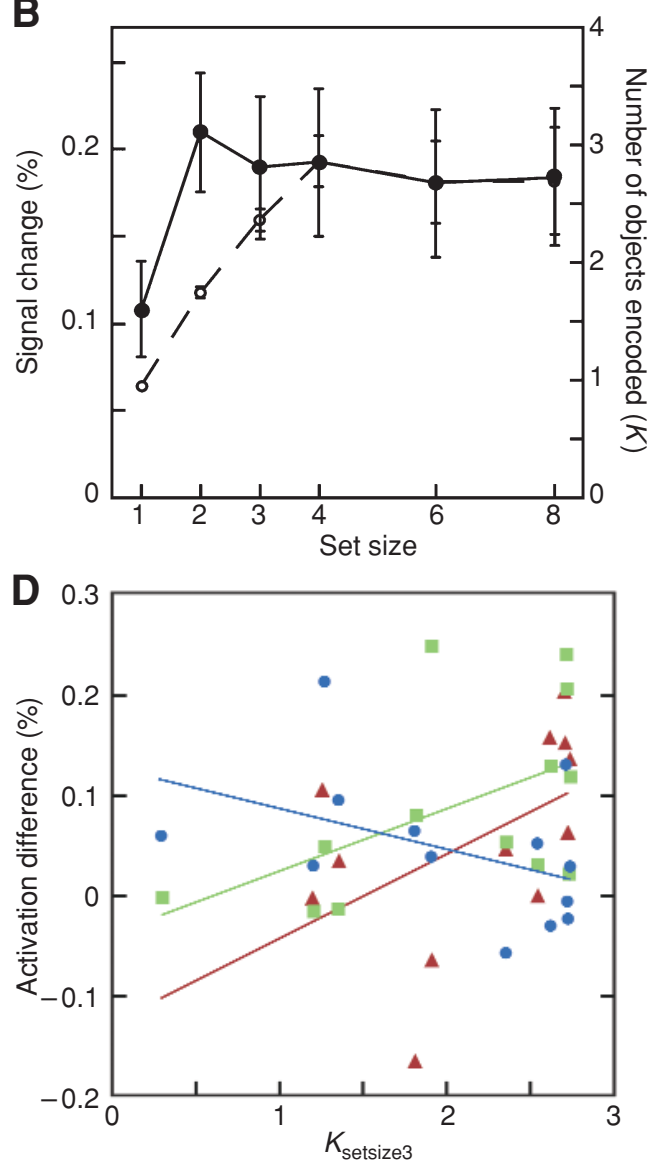

Figure 6. Right posterior middle frontal gyrus (rpMFG) region of interest. (A) The statistical parametric map of group-averaged, $K$-weighted visual short-term memory (VSTM) load function in Experiment 1 reveals activation in the rpMFG (threshold $t=2.12)$. The peak voxel Talairach coordinates are $x, y$, $z,+26,-5,+45$. (B) Behavioral $K$ function (empty circles, dashed line) and rpMFG peak response function (solid circles and line) for the group average analysis in Experiment 1. Percentage of signal change is on the left ordinate; $K$ values are on the right ordinate. Peak percentage of signal change corresponded to the volume acquired $5.6 \mathrm{sec}$ after stimulus presentation. (C) Correlation of individual $K_{\max }$ with the activation difference between set size $K_{\max }$ and set size 1 in Experiment 1. (D) Correlation of individual $K_{\text {setsize3 }}$ with the activation difference between set sizes 3 and 1 for the encoding (red), maintenance (green), and retrieval (blue) phases of VSTM in Experiment 2.

relate with individuals' VSTM capacity $[r(14)=.25$, n.s.; Figure 6C]. Finally, there was no correlation between rpMFG activity and individuals' VSTM capacity at set size 3 for the encoding $[r(10)=.49$, n.s. $]$ and retrieval $[r(11)=-.42$, n.s. $]$ phases of VSTM in Experiment 2 , but there was a marginal correlation during maintenance $[r(11)=.53, p=.06$; Figure 6D]. Thus, both the group average and the individual-differences approaches suggest that rpMFG activity does not tightly correlate with VSTM storage capacity.

\section{DISCUSSION}

Using a voxelwise individual-differences approach, we showed that posterior parietal/superior occipital cortex activity predicts individual differences in VSTM capacity. Importantly, this area largely overlapped with the
PPC region that was isolated with a group-based approach (Todd \& Marois, 2004). This key result suggests that the IPS/IOS does not simply contribute a generic component to VSTM storage capacity, since it may also be involved in setting individual differences in VSTM storage capacity. Thus, just as an individual-differences approach has consolidated the importance and clarified the role of the lateral prefrontal cortex in working memory (Rypma et al., 2002; Rypma \& D'Esposito, 1999, $2000)$, the present individual-differences analysis provides converging evidence with the group average approach (Todd \& Marois, 2004) that the PPC is a key neural locus of our limited capacity for storing a mental representation of the visual world (Todd \& Marois, 2004).

By virtue of its central role in goal-driven attention and spatial working memory (Corbetta, Kincade, \& Shulman, 2002; Corbetta \& Shulman, 2002; LaBar, Gitelman, Par- 
rish, \& Mesulam, 1999; Wojciulik \& Kanwisher, 1999) and in visual feature integration (Friedman-Hill, Robertson, \& Treisman, 1995; Shafritz, Gore, \& Marois, 2002), the PPC is well positioned to build an integrated mental representation of the visual scene. However, it is important to note that the present experimental paradigm focused on capacity limits in storing all of the information present in the visual scene - that is, both the identity (color) and the location of objects. Although object and spatial working memory activate overlapping networks (Haxby, Petit, Ungerleider, \& Courtney, 2000; Mecklinger, Bosch, Gruenewald, Bentin, \& von Cramon, 2000; Postle, Stern, et al., 2000; Sala et al., 2003), the processing of object identity and location information preferentially activates separate ventral inferior temporal and dorsal parietal processing streams, respectively (Courtney, Ungerleider, Keil, \& Haxby, 1996; Haxby et al., 1991; Postle \& D’Esposito, 1999; Postle, Stern, et al., 2000; Ungerleider \& Mishkin, 1982). Furthermore, there is psychophysical and neuropsychological evidence that VSTM stores for spatial information and object information are at least partly distinct (Della Sala, Gray, Baddeley, Allamano, \& Wilson, 1999; Klauer \& Zhao, 2004; Logie, 1995; Logie \& Marchetti, 1991; Mottaghy, Gangitano, Sparing, Krause, \& PascualLeone, 2002; Oliveri et al., 2001; Tresch, Sinnamon, \& Seamon, 1993; Woodman, Vogel, \& Luck, 2004). Thus, although the present study highlights the importance of the posterior parietal/superior occipital cortex in the capacity limit of storing the entire visual scene, it is certainly possible that different brain regions could contribute predominantly to purely object-based forms of VSTM storage capacity.

Although the correlation analyses support the hypothesis that the IPS/IOS is involved in VSTM storage capacity, they also suggest that it is unlikely to be the only brain region contributing to individual differences in VSTM storage capacity. Indeed, IPS/IOS activity accounts for about $40 \%$ of the intersubject variance in VSTM storage capacity. Correspondingly, the ROI-based analyses suggested that individual differences in VSTM capacity may also be reflected in the activity of additional brain regions, albeit not as consistently and robustly as in the IPS/IOS. AC activity was found to be poorly sensitive to individual differences in VSTM capacity, with either the short (Experiment 1) or the long (Experiment 2) retention interval paradigms. These results are generally consistent with the group average results (Todd \& Marois, 2004): Time course analysis of the AC suggests that it is activated later than the IPS/IOS and the VO cortex, primarily at retrieval (Todd \& Marois, 2004). Similarly, other work has shown that AC activity in visual working memory tasks climaxes at retrieval (Petit, Courtney, Ungerleider, \& Haxby, 1998). Together, these results are in line with the idea that the AC cortex is involved primarily in responserelated processes (Carter et al., 1998; Paus, 2001; Smith \& Jonides, 1999; Swick \& Turken, 2002; van Veen, J. D. Cohen, Botvinick, Stenger, \& Carter, 2001; Zhang, Leung, \& Johnson, 2003) and may exert little function in storing the mental representation of the visual scene.
By contrast to the $\mathrm{AC}, \mathrm{VO}$ cortex activity showed an intriguing relationship with individual differences in VSTM capacity. On the one hand, both the group average (Todd \& Marois, 2004) and the individual-differences (present study) analyses of Experiment 1 suggest that the VO cortex activity does not robustly track the number of objects that can be stored in VSTM. Indeed, there is evidence that the VO cortex may be more sensitive to sensory/ perceptual load than to VSTM load, since its activity is modulated by the number of objects present in a scene even when the objects need not be stored in VSTM (Todd \& Marois, 2004). Correspondingly, we observed no significant maintenance-related $\mathrm{VO}$ cortex activity at the group level in a VSTM task with a long retention interval (Todd \& Marois, 2004). On the other hand, the individualdifferences analysis in Experiment 2 (extended retention interval) revealed that $\mathrm{VO}$ cortex activity is correlated with individual differences in VSTM capacity during the maintenance phase of VSTM. Previous studies of ventral stream contributions to VSTM (in either the VO or the inferior temporal cortex) have yielded conflicting results (Druzgal \& D'Esposito, 2003; Jha \& McCarthy, 2000; Leung et al., 2002; Linden et al., 2003; Munk et al., 2002; Pessoa et al., 2002; Sala et al., 2003), with several of these studies showing no evidence of sustained activity across extended retention intervals (Jha \& McCarthy, 2000; Munk et al., 2002; Pessoa et al., 2002). The results of the latter studies, including our own (Todd \& Marois, 2004), suggest that information is not maintained in the ventral stream during VSTM. However, the individualdifferences analysis in Experiment 2 indicates that even when a group approach reveals neither a load effect nor even a sustained response throughout the retention interval of a VSTM task, activation levels in the VO cortex can still contain information about individual differences in VSTM capacity. These results may serve as a cautionary note that simply performing group average analyses of visual working memory load, as is currently performed in most studies, may conceal more subtle neural contributions to VSTM that are revealed with an individual-differences approach.

Our group analysis of VSTM capacity did not reveal any load-sensitive regions in the lateral frontal or the prefrontal cortex, even when the statistical threshold was relaxed tenfold (Todd \& Marois, 2004). This is despite the fact that numerous studies in human and nonhuman primates have shown the importance of the frontal and prefrontal cortex in visual working memory (J. D. Cohen et al., 1997; Courtney et al., 1997; Funahashi et al., 1989; Jha \& McCarthy, 2000; Leung et al., 2002; Miller, Erickson, \& Desimone, 1996; Munk et al., 2002; Postle, Berger, Taich, \& D'Esposito, 2000; Quintana \& Fuster, 1992; Rao, Rainer, \& Miller, 1997). For this reason, we further explored possible contributions of frontal/prefrontal regions to VSTM capacity by analyzing a region of the rpMFG that surfaced in the SPM when the threshold in the group average analysis of VSTM storage capacity (Todd \& Marois, 2004) was relaxed 100-fold (Figure 6A). The location of this rpMFG activation is consistent with 
the findings of several visual working memory studies (Courtney et al., 1998; Jha \& McCarthy, 2000; Leung et al., 2002; Linden et al., 2003; Pessoa et al., 2002). However, the peak response function across set sizes for this brain region revealed a step function that differed markedly from the quadratic behavioral function describing the number of objects stored in VSTM (Figure 6B; Todd \& Marois, 2004). These results strongly suggest that the rpMFG does not store individual representations of visual objects and, hence, is unlikely to represent the site of VSTM storage capacity. This conclusion is further supported by the finding that the rpMFG does not appear to robustly track individual differences in VSTM capacity.

If the rpMFG does not closely track the number of objects stored in VSTM, what might account for its stepfunction with VSTM load? The Talairach coordinates of the rpMFG place it near the human homologue of the frontal eye field (FEF; Paus, 1996), an area involved in controlling eye movements and covert shifts of attention (Corbetta, 1998). Thus, rpMFG activity could reflect either microsaccades, attention shifts, or more generally speaking, attentional deployment during the VSTM task. Interestingly, a similar step-function in the human FEF has also been found in a visual object-tracking experiment in which a parametric manipulation of attentional load was used (Culham, Cavanagh, \& Kanwisher, 2001), lending further credence to the idea that the rpMFG role in VSTM may be attention related. Specifically, the MFG could help maintain attentional focus toward the object representations that are stored in the PPC and the occipital cortex. The involvement of the MFG in VSTM tasks may be particularly important for long retention intervals that require maintenance of attentional focus, which may account for the moderate correlation between this brain region's activity and individual differences in VSTM capacity. Such an attentional function of the frontal/prefrontal cortex in working memory has been proposed by Curtis and D'Esposito (2003).

Thus, although the present MFG results are consistent with the notion that the frontal/prefrontal cortex is involved in VSTM (e.g., J. D. Cohen et al., 1997; Courtney et al., 1998; Courtney et al., 1997; Jha \& McCarthy, 2000; Leung et al., 2002; Linden et al., 2003), they support recent models suggesting that the role of these brain regions in visual working memory does not include storage of visuospatial information per se (Curtis \& D'Esposito, 2003; D'Esposito \& Postle, 1999; Passingham \& Sakai, 2004; Postle, Berger, Goldstein, Curtis, \& D’Esposito, 2001; Rowe, Toni, Josephs, Frackowiak, \& Passingham, 2000). Indeed, these studies are generally consistent with brain lesion work indicating that although the prefrontal cortex may be required for active maintenance of visual information over long delays, it likely does not dictate storage capacity (D'Esposito \& Postle, 1999).

The results of both the group average (Todd \& Marois, 2004) and the individual-differences (present study) analy- ses are highly consistent with a recent electrophysiological study of VSTM storage capacity (Vogel \& Machizawa, 2004). That study demonstrated a strong correlation between amplitude differences of ERPs across memory array sizes and individual differences in VSTM capacity. Furthermore, the ERP signal was strongest at parietal and occipital electrode sites, a position that is consistent with the posterior parietal/occipital foci of activation observed in the present imaging study.

As with the ERP findings, our fMRI study revealed a positive correlation between an individual's behavioral measure of VSTM capacity and the set size at which the neural response reached asymptote. Other working memory studies have shown a positive relationship between performance and neural activity (e.g., Olesen et al., 2004; Pessoa et al., 2002). However, the relationship between brain activity and performance levels is not always positive, since increased task performance has also been associated with decreased brain activity (e.g., Beauchamp, Dagher, Aston, \& Doyon, 2003; Jansma, Ramsey, Slagter, \& Kahn, 2001; Jenkins, Brooks, Nixon, Frackowiak, \& Passingham, 1994; Raichle et al., 1994). Such inverse relationships are typically assumed to reflect more efficient neural processing (e.g., Jansma et al., 2001; Rypma et al., 2002). If so, the positive correlation between PPC activity and individuals' VSTM capacity suggests that increased capacity may not be associated with more efficient neural processing per se. Instead, the storage of additional objects in VSTM is likely to result from either an enhancement of activity of the nerve cells within a given neural tissue or the recruitment of additional cells within the same tissue. In either case, our results suggest that subjects who can store a larger representation of the visual scene may do so because they have a greater neural cache for this representation in the PPC.

\section{REFERENCES}

Alvarez, G. A., \& Cavanagh, P. (2004). The capacity of visual shortterm memory is set both by visual information load and by number of objects. Psychological Science, 15, 106-111.

Baddeley, A. [D.] (1986). Working memory. New York: Oxford University Press.

Baddeley, A. D., \& Logie, R. (1999). Working memory: The multiple component model. In A. Miyake \& P. Shah (Eds.), Models of working memory: Mechanisms of active maintenance and executive control (pp. 28-61). New York: Cambridge University Press.

Beauchamp, M. H., Dagher, A., Aston, J. A., \& Doyon, J. (2003). Dynamic functional changes associated with cognitive skill learning of an adapted version of the Tower of London task. NeuroImage, 20, 1649-1660.

Becker, M. W., Pashler, H., \& Anstis, S. (2000). The role of iconic memory in change-detection tasks. Perception, 29, 273-286.

Bor, D., Duncan, J., Wiseman, R. J., \& Owen, A. M. (2003). Encoding strategies dissociate prefrontal activity from working memory demand. Neuron, 37, 361-367.

Boynton, G. M., Engel, S. A., Glover, G. H., \& Heeger, D. J. (1996). Linear systems analysis of functional magnetic resonance imaging in human V1. Journal of Neuroscience, 16, 4207-4221.

Braver, T. S., Cohen, J. D., Nystrom, L. E., Jonides, J., Smith, E. E., \& Noll, D. C. (1997). A parametric study of prefrontal cortex involvement in human working memory. NeuroImage, 5, 49-62.

Callicott, J. H., Mattay, V. S., Bertolino, A., Finn, K., Coppola, R., 
Frank, J. A., Goldberg, T. E., \& Weinberger, D. R. (1999). Physiological characteristics of capacity constraints in working memory as revealed by functional MRI. Cerebral Cortex, 9, 20-26.

Carter, C. S., Braver, T. S., Barch, D. M., Botvinick, M. M., Noll, D., \& Cohen, J. D. (1998). Anterior cingulate cortex, error detection, and the online monitoring of performance. Science, 280, 747-749.

Chafee, M. V., \& Goldman-RaKic, P. S. (1998). Matching patterns of activity in primate prefrontal area $8 \mathrm{a}$ and parietal area 7ip neurons during a spatial working memory task. Journal of Neurophysiology, 79, 2919-2940.

Chun, M. M., \& Potter, M. C. (1995). A two-stage model for multiple target detection in rapid serial visual presentation. Journal of Experimental Psychology: Human Perception \& Performance, 21, 109127.

Cohen, J., Cohen, P., West, S. G., \& Aiken, L. S. (2003). Applied multiple regression/correlation analysis for the behavioral sciences (3rd ed.). Mahwah, NJ: Erlbaum.

Cohen, J. D., Perlstein, W. M., Braver, T. S., Nystrom, L. E., Noll, D. C., Jonides, J., \& Smith, E. E. (1997). Temporal dynamics of brain activation during a working memory task. Nature, 386, 604-608.

Constantinidis, C., \& Steinmetz, M. A. (1996). Neuronal activity in posterior parietal area 7a during the delay periods of a spatial memory task. Journal of Neurophysiology, 76, 1352-1355.

CorbetTa, M. (1998). Frontoparietal cortical networks for directing attention and the eye to visual locations: Identical, independent, or overlapping neural systems? Proceedings of the National Academy of Sciences, 95, 831-838.

Corbetta, M., Kincade, J. M., \& Shulman, G. L. (2002). Neural systems for visual orienting and their relationships to spatial working memory. Journal of Cognitive Neuroscience, 14, 508-523.

Corbetta, M., \& Shulman, G. L. (2002). Control of goal-directed and stimulus-driven attention in the brain. Nature Reviews Neuroscience, 3, 201-215.

CoRNOLdI, C., \& VeCCHI, T. (2003). Visuo-spatial working memory and individual differences. New York: Psychology Press.

Courtney, S. M., Petit, L., Maisog, J. M., Ungerleider, L. G., \& HAXBY, J. V. (1998). An area specialized for spatial working memory in human frontal cortex. Science, 279, 1347-1351.

Courtney, S. M., Ungerleider, L. G., Keil, K., \& Haxby, J. V. (1996). Object and spatial visual working memory activate separate neural systems in human cortex. Cerebral Cortex, 6, 39-49.

Courtney, S. M., Ungerleider, L. G., Keil, K., \& Haxby, J. V. (1997). Transient and sustained activity in a distributed neural system for human working memory. Nature, 386, 608-611.

CowAN, N. (2001). The magical number 4 in short-term memory: A reconsideration of mental storage capacity. Behavioral \& Brain Sciences, 24, 87-185.

Culham, J. C., Cavanagh, P., \& Kanwisher, N. G. (2001). Attention response functions: Characterizing brain areas using $\mathrm{fMRI}$ activation during parametric variations of attentional load. Neuron, 32, $737-$ 745 .

Curtis, C. E., \& D'Esposito, M. (2003). Persistent activity in the prefrontal cortex during working memory. Trends in Cognitive Sciences, 7, 415-423

Della Sala, S., Gray, C., Baddeley, A., Allamano, N., \& Wilson, L. (1999). Pattern span: A tool for unwelding visuo-spatial memory. Neuropsychologia, 37, 1189-1199.

D'Esposito, M., \& Postle, B. R. (1999). The dependence of span and delayed-response performance on prefrontal cortex. Neuropsychologia, 37, 1303-1315.

Druzgal, T. J., \& D'Esposito, M. (2003). Dissecting contributions of prefrontal cortex and fusiform face area to face working memory. Journal of Cognitive Neuroscience, 15, 771-784.

Duncan, J., Bundesen, C., Olson, A., Humphreys, G., Chavda, S. \& ShibuYa, H. (1999). Systematic analysis of deficits in visual attention. Journal of Experimental Psychology: General, 128, 450-478.

Friedman-Hill, S. R., Robertson, L. C., \& Treisman, A. (1995). Parietal contributions to visual feature binding: Evidence from a patient with bilateral lesions. Science, 269, 853-855.

Funahashi, S., Bruce, C. J., \& Goldman-Rakic, P. S. (1989). Mne- monic coding of visual space in the monkey's dorsolateral prefrontal cortex. Journal of Neurophysiology, 61, 331-349.

FUSTER, J. M. (1990). Inferotemporal units in selective visual attention and short-term memory. Journal of Neurophysiology, 64, 681-697.

GOLDMAN-RAKIC, P. S. (1996). Regional and cellular fractionation of working memory. Proceedings of the National Academy of Sciences, 93, 13473-13480

Haxby, J. V., Grady, C. L., Horwitz, B., Ungerleider, L. G., Mishis, M., Carson, R. E., Herscovitch, P., Schapiro, M. B., \& RAPOPORT, S. I. (1991). Dissociation of object and spatial visual processing pathways in human extrastriate cortex. Proceedings of the National Academy of Sciences, 88, 1621-1625.

Haxby, J. V., Petit, L., Ungerleider, L. G., \& Courtney, S. M. (2000). Distinguishing the functional roles of multiple regions in distributed neural systems for visual working memory. NeuroImage, 11, 145-156.

Jansma, J. M., Ramsey, N. F., Slagter, H. A., \& Kahn, R. S. (2001). Functional anatomical correlates of controlled and automatic processing. Journal of Cognitive Neuroscience, 13, 730-743.

Jenkins, I. H., Brooks, D. J., Nixon, P. D., Frackowiak, R. S. J., \& Passingham, R. E. (1994). Motor sequence learning: A study with positron emission tomography. Journal of Neuroscience, 14, 37753790.

Jha, A. P., \& McCarthy, G. (2000). The influence of memory load upon delay-interval activity in a working-memory task: An eventrelated functional MRI study. Journal of Cognitive Neuroscience, $\mathbf{1 2}$ 90-105.

Joliceur, P., Dell'Acoua, R., \& Crebolder, J. M. (2001). The attentional blink bottleneck. In K. Shapiro (Ed.), The limits of attention: Temporal constraints in human information processing (pp. 8299). New York: Oxford University Press.

Jonides, J., Smith, E. E., Koeppe, R. A., Awh, E., Minoshima, S., \& Mintun, M. A. (1993). Spatial working memory in humans as revealed by PET. Nature, 363, 623-625.

Klauer, K. C., \& Zhao, Z. (2004). Double dissociations in visual and spatial short-term memory. Journal of Experimental Psychology: General, 133, 355-381.

KourtZi, Z., \& KanWISHER, N. (2001). Representation of perceived object shape by the human lateral occipital complex. Science, $\mathbf{2 9 3}$ 1506-1509.

LaBar, K. S., Gitelman, D. R., Parrish, T. B., \& Mesulam, M. (1999). Neuroanatomic overlap of working memory and spatial attention networks: A functional MRI comparison within subjects. NeuroImage, 10, 695-704.

Leung, H. C., Gore, J. C., \& Goldman-Rakic, P. S. (2002). Sustained mnemonic response in the human middle frontal gyrus during online storage of spatial memoranda. Journal of Cognitive Neuroscience, 14, 659-671.

Linden, D. E., Bittner, R. A., Muckli, L., Waltz, J. A., Kriegeskorte, N., Goebel, R., Singer, W., \& MunK, M. H. J. (2003). Cortical capacity constraints for visual working memory: Dissociation of fMRI load effects in a fronto-parietal network. NeuroImage, 20, 1518-1530.

LogIe, R. H. (1995). Visuo-spatial working memory. Hillsdale, NJ: Erlbaum.

Logie, R. H., \& Marchetti, C. (1991). Visuo-spatial working memory: Visual, spatial or central executive? In R. H. Logie \& M. Denis (Eds.), Mental images in human cognition (pp. 105-115). Amsterdam: North-Holland

LucK, S. J., \& Vogel, E. K. (1997). The capacity of visual working memory for features and conjunctions. Nature, 390, 279-281.

Mecklinger, A., Bosch, V., Gruenewald, C., Bentin, S., \& von Cramon, D. Y. (2000). What have Klingon letters and faces in common? An fMRI study on content-specific working memory systems. Human Brain Mapping, 11, 146-161.

Miller, E. K., \& Desimone, R. (1994). Parallel neuronal mechanisms for short-term memory. Science, 263, 520-522.

Miller, E. K., Erickson, C. A., \& Desimone, R. (1996). Neural mechanisms of visual working memory in prefrontal cortex of the macaque. Journal of Neuroscience, 16, 5154-5167. 
Mottaghy, F. M., Gangitano, M., Sparing, R., Krause, B. J., \& Pascual-Leone, A. (2002). Segregation of areas related to visual working memory in the prefrontal cortex revealed by rTMS. Cerebral Cortex, 12, 369-375.

Munk, M. H., Linden, D. E., Muckli, L., Lanfermann, H., Zanella, F. E., Singer, W., \& Goebel, R. (2002). Distributed cortical systems in visual short-term memory revealed by event-related functional magnetic resonance imaging. Cerebral Cortex, 12, 866-876.

Olesen, P. J., Westerberg, H., \& Klingberg, T. (2004). Increased prefrontal and parietal activity after training of working memory. $\mathrm{Na}$ ture Neuroscience, 7, 75-79.

Oliveri, M., Turriziani, P., Carlesimo, G. A., Koch, G., Tomaiuolo, F., Panella, M., \& Caltagirone, C. (2001). Parieto-frontal interactions in visual-object and visual-spatial working memory: Evidence from transcranial magnetic stimulation. Cerebral Cortex, 11, 606618

Pashler, H. (1988). Familiarity and visual change detection. Perception \& Psychophysics, 44, 369-378.

Passingham, D., \& SaKaI, K. (2004). The prefrontal cortex and working memory: Physiology and brain imaging. Current Opinion in Neurobiology, 14, 163-168.

Paulesu, E., Frith, C. D., \& Frackowiak, R. S. (1993). The neural correlates of the verbal component of working memory. Nature, $\mathbf{3 6 2}$ 342-345.

PAus, T. (1996). Location and function of the human frontal eye-field: A selective review. Neuropsychologia, 34, 475-483.

Paus, T. (2001). Primate anterior cingulate cortex: Where motor control, drive and cognition interface. Nature Reviews Neuroscience, $\mathbf{2}$, 417-424.

Pessoa, K., Gutierrez, E., Bandettini, P. A., \& Ungerleider, L. G. (2002). Neural correlates of visual working memory: fMRI amplitude predicts task performance. Neuron, 35, 975-987.

Petit, L., Courtney, S. M., Ungerleider, L. G., \& Haxby, J. V. (1998). Sustained activity in the medial wall during working memory delays. Journal of Neuroscience, 18, 9429-9437.

PhiLlips, W. A. (1974). On the distinction between sensory storage and short-term visual memory. Perception \& Psychophysics, 16, 283290

Postle, B. R., Berger, J. S., \& D’Esposito, M. (1999). Functional neuroanatomical double dissociation of mnemonic and executive control processes contributing to working memory performance. Proceedings of the National Academy of Sciences, 96, 12959-12964.

Postle, B. R., Berger, J. S., Goldstein, J. H., Curtis, C. E., \& D'Esposito, M. (2001). Behavioral and neurophysiological correlates of episodic coding, proactive interference, and list length effects in a running span verbal working memory task. Cognitive, Affective, \& Behavioral Neuroscience, 1, 10-21.

Postle, B. R., Berger, J. S., Taich, A. M., \& D’Esposito, M. (2000). Activity in human frontal cortex associated with spatial working memory and saccadic behavior. Journal of Cognitive Neuroscience, 12, 2-14.

Postle, B. R., \& D'Esposito, M. (1999). "What"-then-"where" in visual working memory: An event-related fMRI study. Journal of Cognitive Neuroscience, 11, 585-597.

Postle, B. R., Stern, C. E., Rosen, B. R., \& Corkin, S. (2000). An fMRI investigation of cortical contributions to spatial and nonspatial visual working memory. NeuroImage, 11, 409-423.

Quintana, J., \& Fuster, J. M. (1992). Mnemonic and predictive functions of cortical neurons in a memory task. NeuroReport, 3, 721-724.

Quintana, J., \& Fuster, J. M. (1999). From perception to action: Temporal integrative functions of prefrontal and parietal neurons. Cerebral Cortex, 9, 213-221.

Raichle, M. E., Fiez, J. A., Videen, T. O., Macleod, A. M., Pardo, J. V., Fox, P. T., \& Petersen, S. E. (1994). Practice-related changes in human brain functional anatomy during nonmotor learning. Cerebral Cortex, 4, 8-26.

RAO, S. C., RAINer, G., \& Miller, E. K. (1997). Integration of what and where in the primate prefrontal cortex. Science, 276, 821-824.
Rensink, R. A. (2002). Change detection. Annual Review of Psychology, 53, 245-277.

Rensink, R. A., O'Regan, J. K., \& Clark, J. J. (1997). To see or not to see: The need for attention to perceive changes in scenes. Psychological Science, 8, 368-373.

Rowe, J. B., \& Passingham, R. E. (2001). Working memory for location and time: Activity in prefrontal area 46 relates to selection rather than maintenance in memory. NeuroImage, 14, 77-86.

Rowe, J. B., Toni, I., Josephs, O., Frackowiak, R. S. J., \& PassingHAM, R. E. (2000). The prefrontal cortex: Response selection or maintenance within working memory? Science, 288, 1656-1660.

Rypma, B., Berger, J. S., \& D'Esposito, M. (2002). The influence of working-memory demand and subject performance on prefrontal cortical activity. Journal of Cognitive Neuroscience, 14, 721-731.

Rypma, B., \& D'Esposito, M. (1999). The roles of prefrontal brain regions in components of working memory: Effects of memory load and individual differences. Proceedings of the National Academy of Sciences, 96, 6558-6563

RyPMA, B., \& D'Esposito, M. (2000). Isolating the neural mechanisms of age-related changes in human working memory. Nature Neuroscience, 3, 509-515.

SaKai, K., Rowe, J. B., \& Passingham, R. E. (2002). Active maintenance in prefrontal area 46 creates distractor-resistant memory. $\mathrm{Na}$ ture Neuroscience, $\mathbf{5}, 479-484$

Sala, J. B., RÄMÄ, P., \& Courtney, S. M. (2003). Functional topography of a distributed neural system for spatial and nonspatial information maintenance in working memory. Neuropsychologia, 41, 341-356.

Shafritz, K. M., Gore, J. C., \& Marois, R. (2002). The role of the parietal cortex in visual feature binding. Proceedings of the National Academy of Sciences, 99, 10917-10922.

Shapiro, K. L., Arnell, K. M., \& Raymond, J. E. (1997). The attentional blink. Trends in Cognitive Sciences, 1, 291-296.

Simons, D. J. (1996). In sight, out of mind: When object representations fail. Psychological Science, 7, 301-305.

SMith, E. E., \& Jonides, J. (1998). Neuroimaging analyses of human working memory. Proceedings of the National Academy of Sciences, 95, 12061-12068

Smith, E. E., \& Jonides, J. (1999). Storage and executive processes in the frontal lobes. Science, 283, 1657-1661.

SPERLING, G. (1960). The information available in brief visual presentations. Psychological Monographs: General \& Applied, 74, 1-29.

Swick, D., \& Turken, A. U. (2002). Dissociation between conflict detection and error monitoring in the human anterior cingulate cortex. Proceedings of the National Academy of Sciences, 99, 16354-16359.

Talairach, J., \& Tournoux, P. (1988). Co-planar stereotaxic atlas of the human brain: 3-Dimensional proportional system. An approach to cerebral imaging (M. Rayport, Trans.). New York: Thieme.

TodD, J. J., FougniE, D., \& MARoIs, R. (in press). Visual short-term memory load suppresses temporo-parietal junction activity and induces inattentional blindness. Psychological Science.

TodD, J. J., \& MaroIs, R. (2004). Capacity limit of visual short-term memory in human posterior parietal cortex. Nature, 428, 751-754.

Tresch, M. C., Sinnamon, H. M., \& Seamon, J. G. (1993). Double dissociation of spatial and object visual memory: Evidence from selective interference in intact human subjects. Neuropsychologia, 31, 211-219.

UngerLeIDER, L. G., \& MishKIN, M. (1982). Two cortical visual systems. In D. J. Ingle, M. A. Goodale, \& R. J. W. Mansfield (Eds.), Analysis of visual behavior (pp. 549-586). Cambridge, MA: MIT Press

van Veen, V., Cohen, J. D., Botvinick, M. M., Stenger, V. A., \& CARTER, C. S. (2001). Anterior cingulate cortex, conflict monitoring, and levels of processing. NeuroImage, 14, 1302-1308.

Vogel, E. K., \& Machizawa, M. G. (2004). Neural activity predicts individual differences in visual working memory capacity. Nature, 428, 748-751.

Vogel, E. K., Woodman, G. F., \& LucK, S. J. (2001). Storage of features, conjunctions and objects in visual working memory. Journal of Experimental Psychology: Human Perception \& Performance, 27, 92-114. 
WARD, B. D. (2000). Simultaneous inference for fMRI data. Available at http://afni.nimh.nih.gov/pub/dist/doc/manuals/AlphaSim.pdf/. WoJCIULIK, E., \& KANwISHER, N. (1999). The generality of parietal involvement in visual attention. Neuron, 23, 747-764.

Woodman, G., Vogel, E., \& LUCK, S. J. (2004). Independent visual working memory stores for object identity and location. Manuscript submitted for publication.

Zarahn, E., Aguirre, G., \& D’Esposito, M. (1997). A trial-based experimental design for fMRI. NeuroImage, 6, 122-138.

Zhang, J. X., Leung, H.-C., \& Johnson, M. K. (2003). Frontal activations associated with accessing and evaluating information in working memory: An fMRI study. NeuroImage, 20, 1531-1539.

\section{NOTE}

1. Whereas the term capacity in Experiment 1 could denote the maximum number of objects the subjects can store, this is not the case for Experiment 2, given that only set sizes 1 and 3 were used. However, a given individual's capacity is not fixed, since it depends on object complexity (Alvarez \& Cavanagh, 2004) and task specifics (e.g., duration of retention interval). Thus, the term capacity in this article refers either to the maximum number of objects one can store (Experiment $1 ; K_{\max }$ ) or to the number of objects one can store at the largest set size tested (Experiment $\left.2 ; K_{\text {setsize }}\right) . K$ refers to the estimated number of objects stored at a given set size and, therefore, varies with set size.

(Manuscript received October 8, 2004;

revision accepted for publication March 31, 2005.) 